\title{
Evaluation of colistin nephrotoxicity and urinary level of kidney injury molecule-1 in hospitalized adult ICU patients
}

\author{
Afsaneh Vazin $^{\circledR}$, Maryam Malek $^{\circledR}, \operatorname{Iman}_{\text {Karimzadeh }}{ }^{*(\mathbb{}}$ \\ Department of Clinical Pharmacy, Faculty of Pharmacy, Shiraz University of Medical Sciences, Shiraz, Iran
}

\section{A R T I C L E I N F O}

Article Type:

Original

\section{Article History:}

Received: 17 November 2019

Accepted: 30 January 2020

Published online: 20 February 2020

Keywords:

Colistin

Nephrotoxicity

Kidney injury molecule-1

\begin{abstract}
A B S T R A C T
Introduction: Colistin is a cationic polypeptide antibiotic used for treatment of gram-negative infections. Nephrotoxicity is one of the most common adverse effects of colistin.

Objectives: To determine the epidemiology of colistin nephrotoxicity and also to compare the changing pattern of kidney injury molecule 1 (KIM-1) in urine with serum creatinine and urine during the treatment with colistin in patients admitted to intensive care unit (ICU).

Patients and Methods: During 13 months, all patients admitted to adult ICUs of two university hospitals without any documented history of acute or chronic kidney diseases receiving at least one week of colistin were included. Required demographic, clinical, and paraclinical data of the study population was collected. Urinary and serum levels of creatinine, urea, sodium, potassium, magnesium, and KIM-1 were measured at six time points including days zero, 3, 5, 7, 10, and 14 of colistin treatment.

Results: Six patients (18.18\%) developed nephrotoxicity during colistin treatment. Nephrotoxicity was resolved without any intervention in three individuals. None of studied demographic, clinical and laboratory characteristics of the patients had a significant association with the incidence of colistin nephrotoxicity. The pattern of KIM-1 urine level during the course of colistin treatment did not differ significantly among patients with and without nephrotoxicity. The accuracy of KIM-1 urine level in detecting colistin nephrotoxicity was significantly lower than that of serum creatinine on days 0 th, $3 \mathrm{rd}$, 5 th and 7 th.

Conclusion: Nephrotoxicity of colistin is a common complication, usually reversible, KIM-1 was not more accurate than serum creatinine or urine in detecting nephrotoxicity of colistin.
\end{abstract}

Implication for health policy/practice/research/medical education:

Nephrotoxicity of colistin is a common complication, which is usually reversible, and can be managed by adjusting the dosage of the drug or replacement with other drugs with lower nephrotoxicity.

Please cite this paper as: Vazin A, Malek M, Karimzadeh I. Evaluation of colistin nephrotoxicity and urinary level of kidney injury molecule-1 in hospitalized adult ICU patients. J Renal Inj Prev. 2020; 9(2): e13. doi: 10.34172/jrip.2020.13.

\section{Introduction}

Colistin (polymyxin E) is a cationic polypeptide antibiotic. It was available for clinical use since $1959(1,2)$. Colistin has a narrow spectrum of antimicrobial activity. Its antimicrobial effect is concentration-dependent $(3,4)$. Colistin is mostly effective against gram-negative multidrug resistant organisms such as Pseudomonas aeruginosa, Acinetobacter baumannii, and Klebsiella pneumonia (5).

Nephrotoxicity is the most common and worrisome adverse effects of colistin. This complication is dosedependent and usually reversible. Permanent kidney damage is rarely seen. Clinical manifestations of colistin nephrotoxicity include decrease in creatinine clearance, proteinuria, cylindruria or oliguria. The incidence of colistin nephrotoxicity has been reported to be as high as $53.5 \%$ (6).

Kidney injury molecule 1 (KIM-1) is a protein that is up regulated by ischemic or toxic injury at epithelial kidney cells in the proximal tubule. The utility of KIM-1 as a marker of kidney injury caused by nephrotoxic agents has been investigated in several experimental models such as cisplatin, gentamicin, vancomycin, furosemide, doxorubicin, tacrolimus, and heavy metals $(7,8)$.

However, clinical data about the role of KIM-1 in detecting kidney injury was limited and inconclusive. 


\section{Objectives}

This study aimed to determine the pattern and associated factors of colistin nephrotoxicity and also assess the changing pattern as well as the accuracy of urine KIM-1 compared with serum and urine creatinine in critically ill patients at two university hospitals in Shiraz, Iran.

\section{Patients and Methods}

Study setting and sample size

During 13 months from November 2016 to December 2017, a cross-sectional, observational study was conducted at four intensive care units (ICUs) of two teaching clinical settings, Namazi and Rajaei hospitals, affiliated to Shiraz University of Medical Sciences. The Medical Ethics Committee of hospitals approved this study and informed consent form was obtained from all patients. Considering $\alpha=0.05$, the statistical power of $80 \%(1-\beta=0.8)$, and incidence of colistin nephrotoxicity in the literature (45$55 \%$ ), the minimum sample size was calculated to be 30 people.

\section{Patient selection}

Patients with the following features were selected: 1) adults (over 18 years of age), 2) individuals, without any confirmed acute kidney injury (AKI) (increased serum creatinine levels equal to or greater than $0.3 \mathrm{mg} / \mathrm{dL}$ in 48 hours; elevated serum creatinine level or more than 1.5 times compared to baseline during the last 7 days; or urine output less than $0.5 \mathrm{~mL} / \mathrm{kg} /$ hour for 6 hours) or chronic kidney disease, (calculated glomerular filtration rate [GFR] less than $60 \mathrm{~mL} / \mathrm{min} / 1 / 73 \mathrm{~m}^{2}$; or history of dialysis or hemodialysis for more than 3 months), and (3) patients who were candidate for colistin (Beacon ${ }^{\circledR}$ Pharmaceuticals Limited, Colistimethate sodium, DCC Vital, Westminster Industrial Estate, England) treatment for at least one week. Documented underlying kidney disease or receiving colistin in the ICU for less than one week due to any reason were considered as exclusion criteria.

\section{Data collection}

The demographic and clinical data of the population studied included age, gender, weight, height, acute physiology and chronic health evaluation (APACHE) II score within the first 24 hours of ICU admission (9), admission diagnosis, dose, duration of treatment, and indication of colistin, co-administered medications (especially potentially nephrotoxicity drugs such as aminoglycosides, amphotericin B and furosemide), possible modalities taken to control the nephrotoxicity of colistin (e.g., dose reduction or discontinuation), and clinical outcome (length of hospital stay and mortality) were collected.

\section{Biochemical and serological measurements}

Serum urea and creatinine were measured at baseline (day
0 ) and then daily during the course of colistin treatment. Serum electrolytes (sodium, potassium and magnesium) were measured according to routine program of hospital. Regarding the fact that nephrotoxicity of colistin mainly occurred during the first 2 weeks of treatment, urinary creatinine level as well as urinary levels of KIM-1 were measured at six time points including days $0,3,5,7$, 10 , and 14. Measurements of serum and urine levels of electrolytes, urea and creatinine were performed using an auto-analyzer in a session on the same day. To determine the level of KIM-1 in the urine, a portion of the urine samples taken from each patient was stored at the earliest opportunity after sampling at $-80^{\circ} \mathrm{C}$. It was measured altogether at the end of the patients' samplings. The KIM1 urine level measurements were performed using the ELISA method by a double-blinded sandwich antibody technique (Bioassay Technology Laboratory, Shanghai, China).

\section{Study endpoints}

Increase in serum creatinine above $2 \mathrm{mg} / \mathrm{dL}$ or a $50 \%$ reduction in calculated creatinine clearance (calculated by Cockcroft-Gault equation) in comparison with baseline value, or a need for renal replacement therapy were defined as colistin nephrotoxicity (1). Besides the above definition for colistin nephrotoxicity, the RIFLE (risk, injury, failure, loss of kidney function and end-stage kidney disease) criteria was also used for the classification of AKI during the course of colistin treatment in the cohort (10). Other indexes in of nephrotoxicity were acute tubular necrosis (fractional excretion of sodium $>2 \%$ or fractional excretion of urea $>50 \%$ in case of co-administration of diuretics), hypokalemia (serum potassium level less than $3 \mathrm{mEq} / \mathrm{L}$ ), hypomagnesemia (serum magnesium level less than $1.2 \mathrm{mEq} / \mathrm{L}$ ), renal potassium wasting (urinary potassium/creatinine $>13 \mathrm{mEq} / \mathrm{g}$ in the presence of hypokalemia), and renal magnesium wasting (fractional excretion of magnesium more than $4 \%$ in the presence of hypomagnesemia) $(11,12)$.

\section{Ethical issues}

The study was conducted in accordance with the Declaration of Helsinki. The protocol was approved by the Ethics Committee of Shiraz University of Medical Sciences (IR.SUMS.REC.1396.594). Informed consent was obtained from all participants before inclusion in the study and they were informed of their right to withdraw from the study at any time. This study was granted by the Shiraz University of Medical Sciences (Grant\# 95-01-10312155).

\section{Statistical analysis}

The study of the normal distribution of continuous variables was performed using Kolmogorov-Smirnov test. Continuous variables with normal and abnormal distribution were expressed as mean \pm standard deviation 
(SD) and median with interquartile range, respectively. In contrast, categorical variables were reported as percentages. The probable relationship between categorical variables was investigated using chi-square or Fisher's exact test. The Fisher's exact test was performed under conditions where more than $20 \%$ of the variables had an expected frequency of less than 5. Parametric and nonparametric continuous variables were analyzed using independent $t$ test and Mann-Whitney $U$ test, respectively. The logistic regression analysis in step-by-step method was used by calculating the odds ratio (OR) and $95 \%$ confidence interval $(95 \% \mathrm{CI})$ to determine the factors associated with colistin nephrotoxicity. Those with $P$ values less than 0.1 were entered into the final multivariate model. Mean values of serum as well as urine creatinine and urine KIM1 at different time points $(0,3 \mathrm{rd}, 5 \mathrm{th}, 7 \mathrm{th}, 10 \mathrm{th}$ and $14 \mathrm{th})$ in each group (with or without nephrotoxicity) alone, as well as between the two groups were compared by oneway analysis of variance with repeated measures. Receiver operating characteristic (ROC) curves were exploited to calculate the area under the curve (AUC) and compare sensitivity and specificity of urine KIM-1 with serum creatinine as well as urine creatinine in the diagnosis of colistin nephrotoxicity at certain time points. Except for univariate logistic regression analysis, $P$ values less than 0.05 were considered to be statistically significant. All descriptive-analytic statistical analysis was performed using IBM SPSS statistics version 20 software.

\section{Results}

During the study period, 60 patients were treated with colistin. Among them, 33 people met the inclusion/ exclusion criteria and entered into the study. In contrast, 27 patients were not eligible for inclusion in the study due to discharge from the hospital or transfer to another ward $(n=11)$, changing the diagnosis or lack of colistin $(n=9)$, or dying before receiving colistin for at least one week $(\mathrm{n}=7)$ (Figure 1).

More than $80 \%$ of the study population were male. The mean \pm SD age of patients was $51.75 \pm 20.88$ years. The highest number of patients was from the ICU of Rajaee hospital (48.48\%), followed by emergency ICU (30.3\%), and general ICU of Namazi hospital (21.21\%). Trauma (57.6\%) was the most common admission diagnosis $(42.4 \%)$. The most frequent indication for the administration of colistin in the cohort was hospitalacquired or ventilator associated pneumonia (81.81\%). The median daily dose of colistin was 6 million units. Fourteen, 12, and 8 patients were received an at least one nephrotoxic agent including vancomycin, furosemide, and aminoglycosides, respectively. Amphotericin B, acyclovir, and calcineurin inhibitors were not given to any patient.

Around 18.18\% (6 patients) developed nephrotoxicity during treatment with colistin. The median time onset of colistin nephrotoxicity was one day. It ranged between 1 and 5 days. Acute tubular necrosis was identified in 27 patients (81.81\%). Based on the RIFLE criteria, 3 and 1 colistin recipients were in the risk and injury categories, respectively.

None of the patients experienced hypocalcemia or hypomagnesemia during colistin treatment. The mean \pm SD of potassium and magnesium supplements administered to patients without colistin nephrotoxicity were $41.11 \pm 36.41$ and $11.85 \pm 7.14 \mathrm{mEq} /$ day, respectively. These values in patients with colistin nephrotoxicity were $50 \pm 10.95$ and $10.66 \pm 8.26 \mathrm{mEq} /$ day, respectively. These differences were not statistically significant $(P=0.294$ and $P=0.723$ for potassium and magnesium daily supplements respectively).

The mean \pm SD hospital stay in patients with and without colistin nephrotoxicity was $63.83 \pm 75.97$ and $61.29 \pm 44.41$ days, respectively. This difference was not statistically significant $(P=0.912)$. The ICU mortality rate did not differ significantly between patients with and

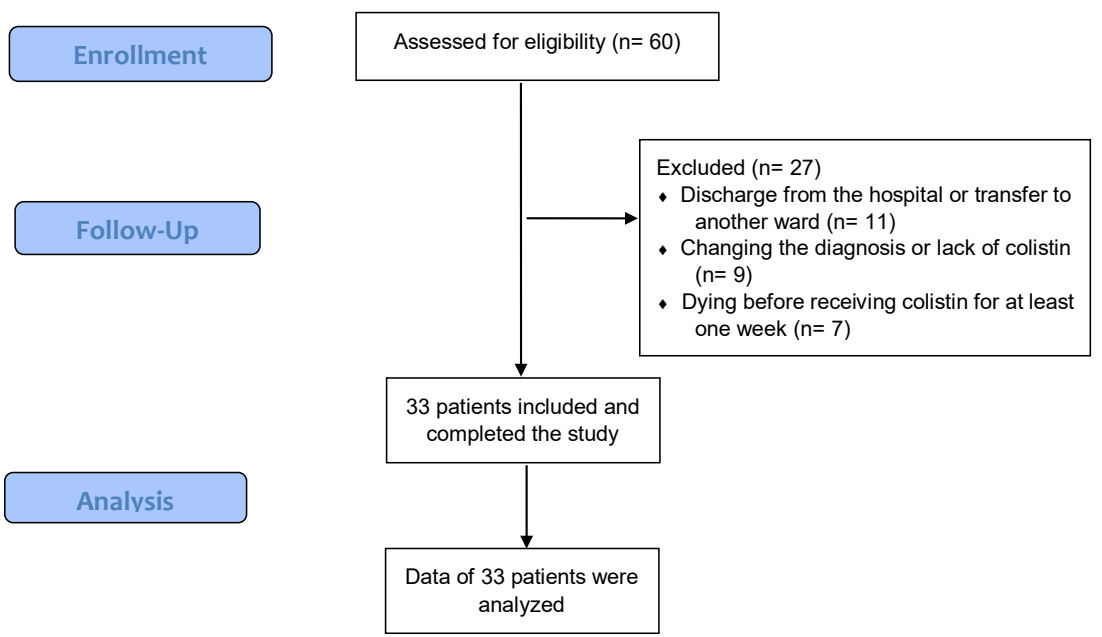

Figure 1. Flow chart of the study. 
without colistin nephrotoxicity $(P=0.137)$. Of six people who developed colistin nephrotoxicity, three patients were treated without any intervention. None of the patients was under hemodialysis or continuous renal replacement therapy due to colistin nephrotoxicity and did not need to discontinue administration of the offending drug.

The mean \pm SD of cumulative dose in patients without nephrotoxicity was higher than those with nephrotoxicity $40.18 \pm 21.86$ million units and $34.33 \pm 16.76$ million units, respectively, however this difference was not statistically significant $(P=0.532)$. According to univariate analysis, age $(\mathrm{OR}=1.057,95 \% \mathrm{CI}=0.999-1.119, P=0.056)$, baseline GFR $(\mathrm{OR}=0.858,95 \% \mathrm{CI}=0.729-1.01, P=0.67)$ and co- administration of furosemide $(\mathrm{OR}=0.07,95 \% \mathrm{CI}=0.007$ $0.707, P=0.024)$ were considered as independent factors of colistin nephrotoxicity. However, multivariate logistic regression analysis demonstrated that none of the demographic, clinical, and laboratory characteristics of the study population had a statistically significant association with the nephrotoxicity of colistin (Table 1).

The trend of changes in serum and urine levels of creatinine and urinary levels of KIM-1 is shown in Figure 2. The overall trend of mean serum creatinine changes within each group, including those experienced or did not experience colistin nephrotoxicity during treatment was statistically significant $(P<0.001)$. The mean changes of

Table 1. Comparison of different demographic and clinical features of the study population $(n=33)$ within patients with and without colistin nephrotoxicity

\begin{tabular}{|c|c|c|c|c|c|c|}
\hline Variable & $\begin{array}{c}\text { Without } \\
\text { nephrotoxicity } \\
(n=27)\end{array}$ & $\begin{array}{c}\text { With } \\
\text { nephrotoxicity } \\
(n=6)\end{array}$ & $\begin{array}{l}\text { Univariate model } \\
\text { OR }(95 \% \mathrm{Cl})\end{array}$ & $P$ value & $\begin{array}{l}\text { Multivariate } \\
\text { model } \\
\text { OR }(95 \% \mathrm{Cl})\end{array}$ & $P$ value \\
\hline \multicolumn{7}{|l|}{ Age (y) } \\
\hline Mean \pm SD & $48.14 \pm 19.98$ & $68 \pm 20.42$ & $1.057(0.999-1.119)$ & 0.056 & $0.97(0.83-1.14)$ & 0.74 \\
\hline Range & 18-86 & $30-85$ & & & & \\
\hline \multicolumn{7}{|l|}{ Gender (\%) } \\
\hline Male & $22(81.48)$ & $5(83.33)$ & $0.88(0.083-9.28)$ & 0.915 & & \\
\hline Female & $5(18.52)$ & $1(16.66)$ & & & & \\
\hline \multicolumn{7}{|c|}{ Baseline GFR (mL/min/1.73 m²) } \\
\hline Mean \pm SD & $124.6 \pm 55.29$ & $93.51 \pm 29.45$ & $0.858(0.729-1.01)$ & 0.067 & $0.86(0.74-1.01)$ & 0.066 \\
\hline Range & $99.48-224.49$ & $87.19-161.87$ & & & & \\
\hline \multicolumn{7}{|l|}{ APACHE II score } \\
\hline Mean \pm SD & $21.4 \pm 4.7$ & $24.5 \pm 7.9$ & $0.965(0.729-1.49)$ & 0.083 & & \\
\hline Range & $19-24$ & 21-28 & & & & \\
\hline \multicolumn{7}{|l|}{ Colistin indication (\%) } \\
\hline Treatment of pneumonia & $22(78.18)$ & $5(84.62)$ & $0.88(0.083-9.28)$ & 0.915 & & \\
\hline Others & $5(12.82)$ & $1(15.38)$ & & & & \\
\hline \multicolumn{7}{|c|}{ Colistin treatment duration (day) } \\
\hline Mean \pm SD & $7.33 \pm 3.93$ & $7.33 \pm 2.25$ & $1(0.782-1.279)$ & 1 & & \\
\hline Range & 3-14 & $5-10$ & & & & \\
\hline \multicolumn{7}{|l|}{ Colistin cumulative dose (mu) } \\
\hline Mean \pm SD & $40.18 \pm 21.86$ & $34.33 \pm 16.76$ & $0.985(0.941-1.032)$ & 0.532 & & \\
\hline Range & $9-90$ & $20-60$ & & & & \\
\hline \multicolumn{7}{|l|}{ Daily dose (mu) } \\
\hline Mean \pm SD & $5.81 \pm 2.58$ & $5.16 \pm 3.06$ & $0.906(0.637-1.28)$ & 0.583 & & \\
\hline Range & 3-9 & $2-9$ & & & & \\
\hline \multicolumn{7}{|l|}{ Loading dose (\%) } \\
\hline Yes & $5(18.51)$ & $3(50)$ & $4.4(0.68-28.6)$ & 0.12 & & \\
\hline No & $22(81.48)$ & $3(50)$ & & & & \\
\hline \multicolumn{7}{|c|}{ Co-administration of vancomycin (\%) } \\
\hline Yes & $12(44.44)$ & $2(33.33)$ & $1.6(0.249-10.27)$ & 0.62 & & \\
\hline No & $15(55.55)$ & $4(66.66)$ & & & & \\
\hline \multicolumn{7}{|c|}{ Co-administration of loop diuretics (furosemide) (\%) } \\
\hline Yes & $7(25.92)$ & $1(16.66)$ & $0.07(0.007-0.707)$ & 0.024 & $0.087(0-118.47)$ & 0.51 \\
\hline No & $20(74.07)$ & $5(83.33)$ & & & & \\
\hline \multicolumn{7}{|c|}{ Co-administration of aminoglycoside (\%) } \\
\hline Yes & $19(70.37)$ & $0(0)$ & & 0.99 & & \\
\hline No & $8(29.63)$ & $6(100)$ & & & & \\
\hline \multicolumn{7}{|l|}{ Final diagnosis (\%) } \\
\hline Trauma & $16(59.26)$ & $3(50)$ & $0.69(0.12-4.05)$ & 0.68 & & \\
\hline Others & $11(40.74)$ & $3(50)$ & & & & \\
\hline
\end{tabular}

Abbreviations: APACHE II, Acute Physiology and Chronic Health Evaluation; mu, million unit; GFR, glomerular filtration rate. 
(A)

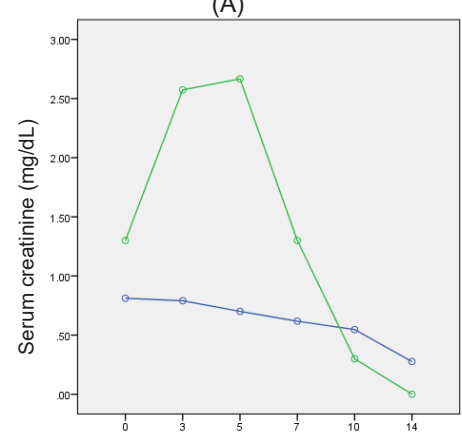

Time of measurement (day)
(B)

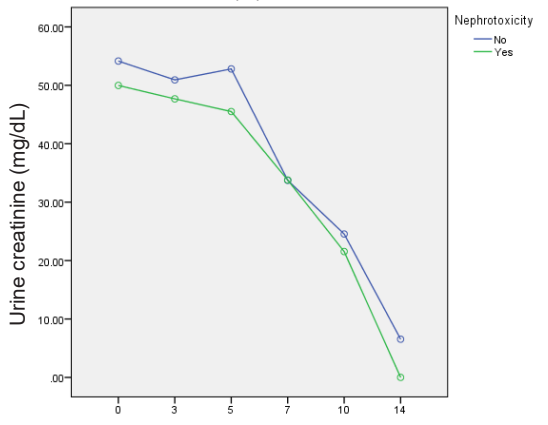

Time of measurement (day)
(C)

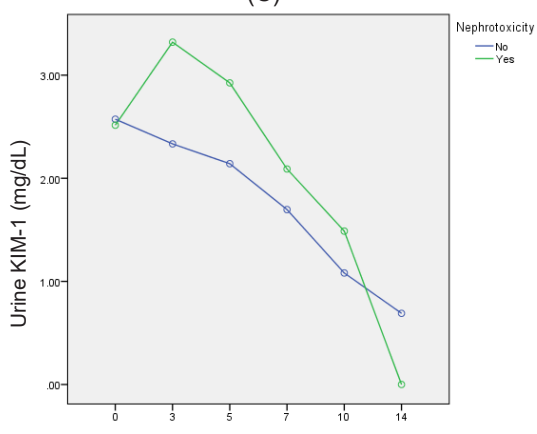

Time of measurement (day)

Figure 2. The changing pattern of serum creatinine (A), urine creatinine (B), and urine KIM-1 (C) at Days $0,3,5,7,10$, and 14 of colistin treatment in patients with and without colistin nephrotoxicity.

serum creatinine between two groups were also statistically significant $(P<0.001)$. The difference in mean values of serum creatinine between the two groups is $1.026 \mathrm{mg} / \mathrm{dL}$ with the $95 \%$ confidence interval from 0.704 to 1.349 .

The trend of changes in urinary creatinine level during the course of colistin treatment within each group with or without nephrotoxicity was statistically significant $(P<0.001)$. In contrast, there was no significant difference between two groups in terms of changes in urinary creatinine level throughout the duration of the study $(P=0.753)$. The mean $(95 \%$ confidence interval) difference in urinary creatinine level between the two groups was $0.431(-22.14$ to $30.21 \mathrm{mg} / \mathrm{dL})$. The overall changes in KIM-1 urine level during colistin treatment in either groups with or without colistin nephrotoxicity was statistically significant $(P<0.001)$; on the other hand, there was no significant difference regarding urinary level of KIM-1 within the course of colistin treatment between two groups $(P=0.402)$. The mean difference in KIM-1 urinary levels in both groups was $0.304 \mathrm{ng} / \mathrm{dL}$ with the $95 \%$ confidence interval from -0.431 to $-1.039 \mathrm{ng} / \mathrm{dL}$.

ROC curves showed that AUC ( $95 \%$ confidence interval) for serum creatinine on days 0 (0.98 [0.93-1], $P<0.001), 3$ (0.97 [0.9-1], $P<0.001), 5$ (1 [1-1], $P<0.001)$, and 7 (0.84 [0.55-1], $P=0.02)$ of colistin treatment were significantly higher than that for urinary creatinine and urinary KIM1. In other words, the sensitivity and specificity (accuracy as a result) of serum creatinine levels in these days in the detection of colistin nephrotoxicity was significantly higher than that of urine KIM-1 as well as urine creatinine
(Figure 3 and Table 2). Regarding the fact that urinary specimens were available for only 11 patients on 10 and 5 patients on day 14 of colistin treatment, the AUC of studied renal biomarkers was not calculated on days 10 and 14 of colistin treatment.

\section{Discussion}

Colistin is not a widely used antibiotic and somewhat a last-resort antibiotic in clinical practice, however, a rise in nosocomial multidrug resistant infections worldwide has increased its use within the recent decades. Nephrotoxicity is a concern for this drug. This study tries to give a pattern of this issue.

The rate of colistin nephrotoxicity in our study was $18.18 \%$, which is within the range that has been reported from the literature $(0-53.5 \%)$ (6) This variation in the frequency of colistin nephrotoxicity can be partially justified by different definitions for colistin nephrotoxicity, different study methodologies, clinical settings, and risk factors. A retrospective study by Pogue et al over a 5-year period in the United States demonstrated that $43 \%$ of patients developed colistin nephrotoxicity (defined by the RIFLE criteria) (13). This rate was $31.9 \%$ in a case-control study in the South Korea (14). The definition of colistin nephrotoxicity used in the present study was according to a relevant review article (15).

Mostly, drug-induced nephrotoxicity is reported as a rapid onset phenomenon. In a study by DeRyke et al, all patients developed nephrotoxicity within the first 5 days of colistin treatment (16) In another study by Pogue et

Table 2. Comparison of area under the curve (95\% confidence interval) of serum creatinine, urine creatinine, and urine KIM-1 at days $0,3,5$, and 7 of colistin treatment in detecting colistin nephrotoxicity

\begin{tabular}{|c|c|c|c|c|c|c|c|c|}
\hline \multirow{2}{*}{ Variable } & \multicolumn{2}{|l|}{ Day 0} & \multicolumn{2}{|l|}{ Day 3} & \multicolumn{2}{|c|}{ Day 5} & \multicolumn{2}{|l|}{ Day 7} \\
\hline & AUC $(95 \% \mathrm{Cl})$ & $P$ value & AUC (95\%CI) & $P$ value & AUC $(95 \% \mathrm{Cl})$ & $P$ value & $\operatorname{AUC}(95 \% \mathrm{Cl})$ & $P$ value \\
\hline Serum creatinine & $0.98(0.93-1)$ & $<0.001$ & $0.97(0.9-1)$ & $<0.001$ & $1(1-1)$ & $<0.001$ & $0.84(0.55-1)$ & 0.02 \\
\hline Urine creatinine & $0.26(0.0-0.54)$ & 0.072 & $0.44(0.19-0.7)$ & 0.67 & $0.42(0.21-0.64)$ & 0.57 & $0.64(0.36-0.92)$ & 0.32 \\
\hline Urine KIM-1 & $0.58(0.32-0.85)$ & 0.51 & $0.58(0.35-0.81)$ & 0.54 & $0.7(0.5-0.93)$ & 0.11 & $0.66(0.39-0.92)$ & 0.28 \\
\hline
\end{tabular}



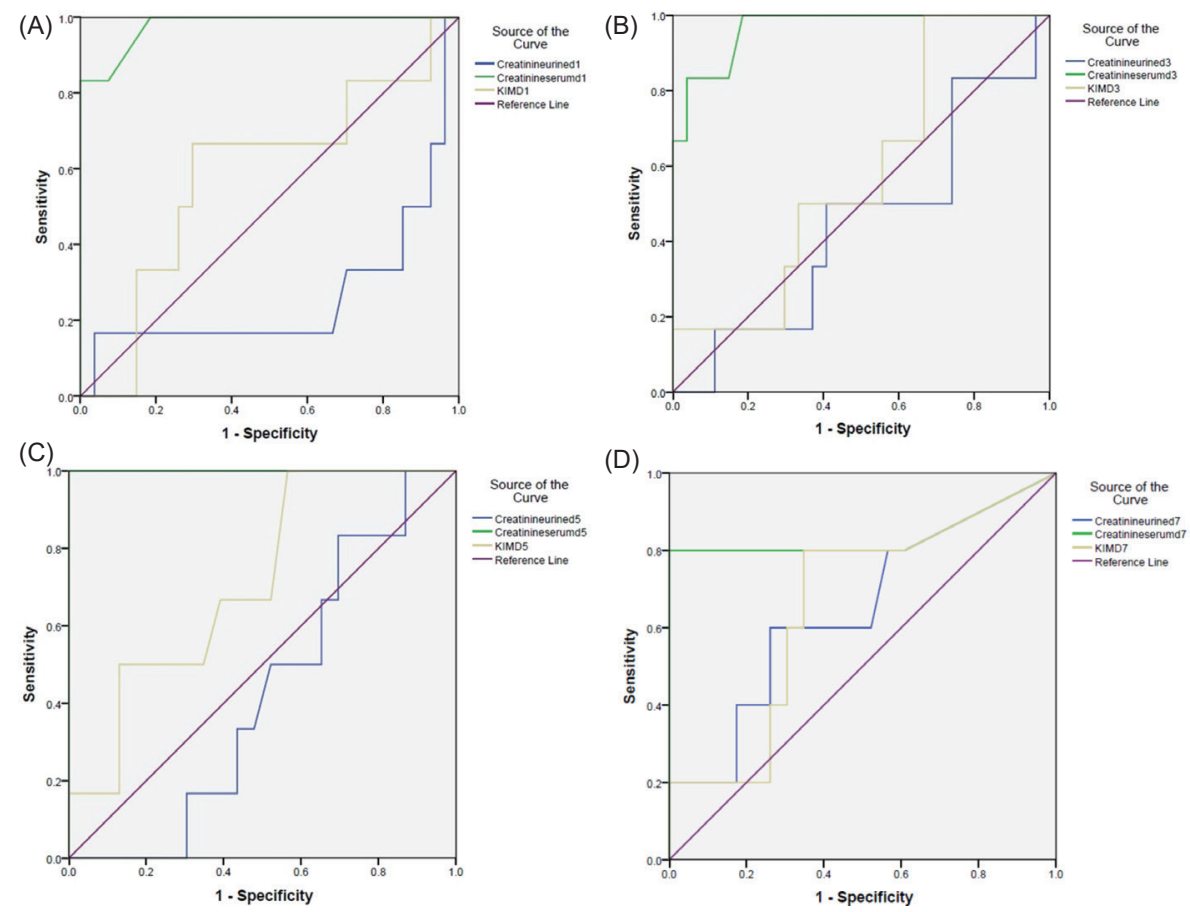

Figure 3. Receiver operating characteristic curves of sensitivity and specificity of serum creatinine, urine creatinine, and urine KIM-1 at days 0 ( $A$ ); 3 (B); 5 (C) and 7 (D) of colistin treatment for detection of nephrotoxicity.

al, the majority of colistin nephrotoxicity $(78 \%)$ occurred during the first week of treatment (13). In a retrospective study by $\mathrm{Ku}$ et al, AKI with colistin occurred in $54.6 \%$ of patients; about $70 \%$ at early treatment (within 7 days) and the remaining at the end of treatment (after 7 days) (17). Patients with early AKI have a higher mortality rate than patients with later AKI. The median time of onset of nephrotoxicity caused by colistin in our study was one day, comparable to the results from the aforementioned studies.

Colistin nephrotoxicity appears to be dose-dependent and reversible. In our study, the mean \pm SD of cumulative dose in patients without nephrotoxicity was higher than those with nephrotoxicity, although this difference was not statistically significant. Similar findings were also observed by Doshi et al in a retrospective study of 49 ICU adult patients received intravenous colistin for at least 48 hours (18). However, in another study by Pogue et al, colistin dose of $\geq 5.0 \mathrm{mg} / \mathrm{kg} / \mathrm{d}$ was an independent predictor for nephrotoxicity (13). At least three other studies also reported colistin daily or its cumulative dose as an independent risk factor for colistin nephrotoxicity $(16,19,20)$. The definite reason for these controversies was unknown.

In the current study, co-administration of furosemide was considered as an independent factor of colistin nephrotoxicity (univariate analysis), however this statistically significant association was not observed in multivariate regression logistics analysis. Similarly, Pogue et al demonstrated a significant association $(\mathrm{OR}=3.0$,
95\% CI: [1.4-6.3], $P=0.004)$ between co-administration of loop diuretics and colistin nephrotoxicity based on bivariate analysis. However, multivariate analysis did not show this association (13). The risk of developing colistin nephrotoxicity was calculated to be 6.5 times higher in patients received at least two concomitant nephrotoxic agents compared with no other nephrotoxic agents (18). Another study identified concomitant use of nonsteroidal anti-inflammatory drugs (NSAIDs) as an independent risk factor for colistin nephrotoxicity (14), however no patient in our study was given NSAIDs.

Regarding clinical outcome in the present study, ICU mortality rate and duration of hospital stay were comparable between patients with and without colistin nephrotoxicity. In addition, half of patients developed colistin nephrotoxicity recovered without any intervention. Pogue et al reported that $30 \%$ of colistin nephrotoxicity resolved within 2 weeks after discontinuing colistin (13). Mortality rate and duration of hospital stay as indexes of clinical outcome have not been considered in relevant studies.

In our study, there was no significant difference regarding urinary level of KIM-1 changes within the course of colistin treatment between patients with and without colistin nephrotoxicity. In addition, the sensitivity and specificity (and overall, accuracy) of KIM1 levels on days $0,3 \mathrm{rd}, 5 \mathrm{th}$, and 7 th of colistin treatment was significantly lower than the serum creatinine level in the detection of colistin nephrotoxicity. So far, six clinical studies have examined the role of urinary levels 
of KIM-1 in detecting nephrotoxicity of drugs including cisplatin, gentamicin, amphotericin $\mathrm{B}$, and vancomycin (21-26). Accordingly, in two studies in pediatric patients, KIM-1 was superior to n-acetyl-beta-glucose amines (NAG), urinary neutrophil gelatinase-associated lipocalin (NGAL), and interleukin-18 in detecting nephrotoxicity caused by cisplatin, ifosfamide, and aminoglycosides (17, 21). Shinke et al studied biomarkers of renal function, including KIM-1, protein chemoattractant monocytes-1 (MCP-1), NAG, NAGL and $\beta 2$-microglubulin, in patients with lung cancer received cisplatin. The values of urinary KIM-1 and MCP-1 (but not NAGL, NAG and $\beta 2$ microglobulin levels) were significantly higher than in patients with AKI caused by cisplatin than those without AKI. They also reported that urinary levels of KIM-1 and MCP-1 were significantly more accurate in detecting AKI in patients received cisplatin compared with other biological markers (23). In contrast to the above data, at least a randomized controlled trial on contrast-inducedAKI to prevent hydration regimes failed to show any significant association between contrast-induced-AKI and urinary KIM-1/urine creatinine ration as well as NGAL/ urine creatinine ratio samples at 4-6 and 48-96 hours after contrast enhanced computed tomography (27). Negative findings in our current study can be partially due to the small sample size (lack of enough statistical power to determine the differences), a limited number of urinary KIM-1 measurements (only at six time points) and not using a gold standard method (such as an exogenous agent such as inulin) to determine GFR and colistin nephrotoxicity. The considerable rate of ATN (81.81\%) in our population rejected the hypothesis that no significant increase in KIM-1 in the urine may be due to the absence of ATN. Noting that KIM-1 is a proximal tubular ischemic index $(7,8)$.

Among antibiotics, including aminoglycosides and amphotericin, electrolyte disturbances such as hypokalemia and hypomagnesemia are common. Hypokalemia and severe hypomagnesemia associated with drug use can lead to metabolic complications, rhabdomyolysis, and life-threatening arrhythmias (28). In the present study, patients with hypokalemia and hypomagnesemia have not been diagnosed. In addition, renal potassium wasting and also renal magnesium wasting were not observed in our study population during treatment with colistin. Similarly, these electrolyte disturbances were not reported or taken into account in relevant clinical studies about colistin nephrotoxicity $(13,14,16,18-20)$.

\section{Conclusion}

Our data demonstrated that less than one-fifth (18.18\%) of colistin recipients developed nephrotoxicity with the median time onset of one day. The mortality rate as well as length of hospital stay did not differ significantly between those with and without colistin nephrotoxicity. None of the patients was under hemodialysis or continuous renal replacement therapy due to colistin nephrotoxicity and did not need to discontinue administration of the offending drug. None of studied demographic, clinical and laboratory characteristics of the patients had a significant association with colistin nephrotoxicity. The accuracy of KIM-1 urine level in detecting colistin nephrotoxicity was significantly lower than that of serum creatinine on days $0,3 \mathrm{rd}, 5 \mathrm{th}$, and 7 th of colistin treatment. Considering the aforementioned limitations of the current study, it is prudent to interpret our findings with caution. Performing a clinical study in a large sample of patients (with both critical and non-critical conditions) along with assessment of KIM-1 urine level more frequently and at close time points seems necessary.

\section{Limitations of the study}

The first limitation of the study is the relative low sample size as well as statistical power and the likelihood of type II error. This is largely due to the application of multiple exclusion criteria. Second, the limited frequency of sampling and KIM-1 urine measurements (only six times) may interfere with defining and depicting the true trend of KIM-1 variations within the course of colistin treatment. Another worrisome issue is the degradation of the kidney biomarkers, especially KIM-1, during storage at $-80^{\circ} \mathrm{C}$ in the freezer, since the measurement of renal biomarkers was postponed until the completion of the sampling process. The package insert within the KIM-1 kit used in the current study has not mentioned this issue. However, Pennemans et al showed that if urine specimens were frozen to measure KIM- 1 within 3 hours of urination, samples were stable for 1.5 years at $-80^{\circ} \mathrm{C}(29)$. Similarly, another study reported that urine specimens were rapidly transported to $4^{\circ} \mathrm{C}$ and then frozen at $-80^{\circ} \mathrm{C}$ for a maximum of 2 days, the urinary KIM-1 will remain stable for 6 months (30). Therefore, the potential undesirable effect of storage conditions (up to 13 months at $-80^{\circ} \mathrm{C}$ ) on the KIM -1 urinary level seems unlikely.

\section{Authors' contribution}

AV contributed to study design and critical review of the manuscript. IK contributed to study design, statistical analyses, and critical review of the manuscript. MM contributed to data collection, patient sampling, biochemical as well as serological measurements, statistical analyses, and manuscript drafting. All authors have read and approved the manuscript.

\section{Conflicts of interest}

The authors report no conflicts of interest regarding this study.

Ethical considerations

Ethical issues (including plagiarism, misconduct, 
data fabrication, falsification, double publication or submission, redundancy) have been completely observed by the authors.

\section{Funding/Support}

This study was financially granted by the Vice-chancellor for Research Affairs of Shiraz University of Medical Sciences. Maryam Malek performed this research as her PharmD project to fulfill the requirements for certification as a pharmacist. The present article was adopted from the proposal number 95-01-103-12155 approved by the vice-chancellor for research affairs of Shiraz University of Medical Sciences.

\section{References}

1. Nation RL, Li JJ. Colistin in the 21st century. Curr Opin Infect Dis.2009;22:535-43.doi:10.1097/QCO.0b013e328332e672.

2. Dhariwal A, Tullu M. Colistin: re-emergence of the 'forgotten' antimicrobial agent. J Postgrad Med. 2013;59:208. doi: $\quad 10.4103 / 0022-3859.118040$.

3. Michalopoulos AS, Karatza DC. Multidrug-resistant gramnegative infections: the use of colistin. Expert Rev Anti Infect Ther. 2010;8:1009-17. doi: 10.1586/eri.10.88.

4. Kwa A, Kasiakou SK, Tam VH, Falagas ME. Polymyxin B: similarities to and differences from colistin (polymyxin E). Expert Rev Anti Infect Ther. 2007;5:811-21. doi: 10.1586/14787210.5.5.811.

5. Gurjar M. Colistin for lung infection: an update. J Intensive Care. 2015;3:3. doi: 10.1186/s40560-015-0072-9.

6. Spapen H, Jacobs R, Van Gorp V, Troubleyn J, Honoré PM. Renal and neurological side effects of colistin in critically ill patients. Ann Intensive Care. 2011;1:14. doi: 10.1186/21105820-1-14.

7. Pianta TJ, Buckley NA, Peake PW, Endre ZH. Clinical use of biomarkers for toxicant-induced acute kidney injury. Biomark Med. 2013;7:441-56. doi: 10.2217/bmm.13.51.

8. Mohamed F, Endre ZH, Buckley NAJ. Role of biomarkers of nephrotoxic acute kidney injury in deliberate poisoning and envenomation in less developed countries. Br J Clin Pharmacol. 2015;80(1):3-19. doi: 10.1111/bcp.12601.

9. Knaus WA, Draper EA, Wagner DP, Zimmerman JE. APACHE II: a severity of disease classification system. Crit Care Med. 1985;13:818-29.

10. Bellomo RJ. Acute Dialysis Quality Initiative workgroup. Acute renal failure-definition, outcome measures, animal models, fluid therapy and information technology needs: the Second International Consensus Conference of the Acute Dialysis Quality Initiative (ADQI) Group. Crit Care. 2004;8:R204-R12.

11. Mount DB. Treatment and prevention of hyperkalemia in adults. Waltham (MA): UpToDate; 2013.

12. Elisaf M, Panteli K, Theodorou J, Siamopoulos K. Fractional excretion of magnesium in normal subjects and in patients with hypomagnesemia. Magnes Res. 1997;10:315-20.

13. Pogue JM, Lee J, Marchaim D, Yee V, Zhao JJ, Chopra T, et al. Incidence of and risk factors for colistin-associated nephrotoxicity in a large academic health system. Clin Infect Dis. 2011;53:879-84. doi: 10.1093/cid/cir611.

14. Kim J, Lee K-H, Yoo S, Pai H. Clinical characteristics and risk factors of colistin-induced nephrotoxicity. Int J Antimicrob Agents. 2009;34:434-8. doi: 10.1016/j. ijantimicag.2009.06.028.

15. Florescu DF, Qiu F, McCartan MA, Mindru C, Fey PD, Kalil AC. What is the efficacy and safety of colistin for the treatment of ventilator-associated pneumonia? A systematic review and meta-regression. Clin Infect Dis. 2012;54:67080. doi: 10.1093/cid/cir934.

16. DeRyke CA, Crawford AJ, Uddin N, Wallace MR. Colistin dosing and nephrotoxicity in a large community teaching hospital. Antimicrob Agents Chemother. 2010;54:4503-5. doi: 10.1128/AAC.01707-09.

17. Ku K, Pogue JM, Moshos J, Bheemreddy S, Wang Y, Bhargava A, et al. Retrospective evaluation of colistin versus tigecycline for the treatment of Acinetobacter baumannii and/or carbapenem-resistant Enterobacteriaceae infections. Am J Infect Control. 2012;40:983-7. doi: 10.1016/j. ajic.2011.12.014.

18. Doshi NM, Mount KL, Murphy CV. Nephrotoxicity associated with intravenous colistin in critically ill patients. Hum Gene Ther Clin Dev. 2011;31:1257-64. doi: 10.1592/ phco.31.12.1257.

19. Rattanaumpawan P, Ungprasert P, Thamlikitkul V. Risk factors for colistin-associated nephrotoxicity. J Infect. 2011;62:187-90. doi: 10.1016/j.jinf.2010.11.013.

20. Hartzell JD, Neff R, Ake J, Howard R, Olson S, Paolino $\mathrm{K}$, et al. Nephrotoxicity associated with intravenous colistin (colistimethate sodium) treatment at a tertiary care medical center. Clin Infect Dis. 2009;48:1724-8. doi: $10.1086 / 599225$.

21. McWilliam SJ, Antoine DJ, Sabbisetti V, Turner MA, Farragher T, Bonventre JV, et al. Mechanism-based urinary biomarkers to identify the potential for aminoglycosideinduced nephrotoxicity in premature neonates: a proof-ofconcept study. PLoS One. 2012;7(8):e43809. doi: 10.1371/ journal.pone.0043809.

22. Piccioni M, Al-Ismaili Z, Devarajan PJ. Biomarkers of cisplatin and ifosfamide nephrotoxicity in children. J Am Soc Nephrol. 2011;22:360A.

23. Shinke H, Masuda S, Togashi Y, Ikemi Y, Ozawa A, Sato $\mathrm{T}$, et al. Urinary kidney injury molecule-1 and monocyte chemotactic protein-1 are noninvasive biomarkers of cisplatin-induced nephrotoxicity in lung cancer patients. Cancer Chemother Pharmacol. 2015;76:989-96. doi: 10.1007/s00280-015-2880-y.

24. George B, Wen X, Mercke N, Gomez M, O’Bryant C, Bowles DW, et al. Profiling of kidney injury biomarkers in patients receiving cisplatin: time-dependent changes in the absence of clinical nephrotoxicity. Clin Pharmacol Ther. 2017;101510-8. doi: 10.1002/cpt.606.

25. Luo Q-H, Chen M-L, Chen Z-L, Huang C, Cheng A-C, Fang J, et al. Evaluation of KIM-1 and NGAL as early indicators for assessment of gentamycin-induced nephrotoxicity in vivo and in vitro. Kidney Blood Press Res. 2016;41:911-8. doi: 10.1159/000452592.

26. McWilliam SJ, Antoine DJ, Jorgensen AL, Smyth RL, Pirmohamed M. Urinary biomarkers of aminoglycosideinduced nephrotoxicity in cystic fibrosis: kidney injury molecule-1 and neutrophil gelatinase-associated lipocalin. Sci Rep. 2018;8(1):5094. doi: 10.1038/s41598-018-23466-4. 
27. Kooiman J, van de Peppel WR, Sijpkens YW, Brulez HF, de Vries PM, Nicolaie MA, et al. No increase in Kidney Injury molecule-1 and neutrophil gelatinase-associated lipocalin excretion following intravenous contrast enhanced-CT. Eur Radiol. 2015;25:1926-34. doi: 10.1007/s00330-015-3624-4.

28. Wazny LD, Brophy DF. Amiloride for the prevention of amphotericin B-induced hypokalemia and hypomagnesemia. Ann Pharmacother. 2000;34:94-7. doi: 10.1345/aph.19127.
29. Pennemans V, Rigo J-M, Penders J, Swennen Q. Collection and storage requirements for urinary kidney injury molecule-1 (KIM-1) measurements in humans. Clin Chem Lab Med. 2012;50:539-43. doi: 10.1515/cclm.2011.796.

30. van de Vrie M, Deegens JK, van der Vlag J, Hilbrands LB. Effect of long-term storage of urine samples on measurement of kidney injury molecule 1 (KIM-1) and neutrophil gelatinase-associated lipocalin (NGAL). Am J Kidney Dis. 2014;63:573-6. doi: 10.1053/j.ajkd.2013.10.010.

Copyright $(2020$ The Author(s); Published by Nickan Research Institute. This is an open-access article distributed under the terms of the Creative Commons Attribution License (http://creativecommons.org/licenses/by/4.0), which permits unrestricted use, distribution, and reproduction in any medium, provided the original work is properly cited. 